\title{
Harnessing class II histone deacetylases in macrophages to combat breast cancer
}

\author{
Hadar Reichman and Ariel Munitz \\ Cellular \& Molecular Immunology (2017) 14, 575-577; doi:10.1038/cmi.2017.32; published online 29 May 2017
}

$\mathrm{V}$ iewing the tumor microenvironment (TME) as a critical factor in cancer biology has been a central paradigm shift in the field in the past two decades. ${ }^{1}$ During this time, the roles of tumor-infiltrating immune cells have gradually been elucidated. The importance of the immune system in the TME is best exemplified by the promising approaches that enhance therapeutic anti-tumor immunity through tumor vaccines, $\mathrm{T}$ cells engineered with a tumor-specific chimeric antigen receptor (CAR), and the blockade of immune checkpoints. ${ }^{2}$ Notably, current antitumorigenic immunotherapies are mostly directed at enhancing the cytotoxic potential of the adaptive immune system targeting cells, such as cytotoxic $\mathrm{CD}^{+} \mathrm{T}$ cells. Nonetheless, the immune response within the tumor microenvironment is an extremely complex system that includes multiple types of immune cells from both the adaptive and the innate immune systems. Thus, therapeutic targeting of additional cells such as macrophages, which are considered the 'hallmark' cells of the innate immune system, might provide added benefits in

Department of Clinical Microbiology and Immunology, The Sackler School of Medicine, Tel-Aviv University, Ramat Aviv 69978, Israel.

Correspondence: Professor A Munitz, PhD, Department of Clinical Microbiology and Immunology, The Sackler School of Medicine, Tel-Aviv University, Ramat Aviv 69978, Israel.

E-mail: arielm@post.tau.ac.il

Received: 9 April 2017; Accepted: 9 April 2017 reducing and eradicating tumors. Macrophages are key cells in the clearance of pathogens and can induce substantial tissue damage. Tumor-associated macrophages are often thought to be cells that promote cell growth and display protumorigenic activities. In fact, "tumorassociated" macrophages can support tumor growth through various mechanisms. For example, they support neoplastic transformation, suppress cytotoxic activity of lymphocytes, promote angiogenesis through secretion of vascular endothelial growth factor and facilitate metastasis. ${ }^{3}$

In recent years, various strategies targeting tumor-associated macrophages have emerged. For example, limiting macrophage development and recruitment has been attempted by targeting macrophage growth factors and chemokines, such as the CSF-1:CSF-1 receptor and CCL2-CCR2 axis. $^{3}$ An alternative approach to replace macrophage depletion strategies could be based on the fact that tumor-associated macrophages preserve their plasticity, which provides therapeutic potential to re-program them from tumor-promoting cells to cells with tumoricidal activities. In a recent study, Guerriero et al. ${ }^{4}$ provided further data supporting the notion that rendering tumor-associated macrophages to display anti-tumorigenic activities may be beneficial. Until recently, the therapeutic potential of class IIa histone deacetylases (HDAC) (for example, HDAC4, HDAC5, HDAC7 and HDAC9) was impaired by the lack of potent and selective compounds that can antagonize their function. Recently, Lobera et al. ${ }^{5}$ described TMP195, which is the most potent and selective class IIa HDAC inhibitor identified to date, with IC50s of 59, 60, 26 and $15 \mathrm{nM}$ for HDAC4, HDAC5, HDAC7 and HDAC9, respectively. Remarkably, monocytes were transcriptionally sensitive to treatment with TMP195, while lymphocytes showed minimal responses. ${ }^{5}$ Moreover, when tested in vitro, TMP195 treatment polarized macrophages into a type 1 proinflammatory phenotype. These results provided the rationale to test the hypothesis that class IIa HDAC inhibition will promote an anti-tumor innate immune response. To this end, Guerriero et al. ${ }^{4}$ used TMP195 in vivo using a macrophage-dependent mouse model, namely, the MMTV-PyMT mammary carcinoma. $^{6}$

Tumor-bearing mice treated with TMP195 displayed increased levels of CD11b+ myeloid cells, specifically in macrophages. The myeloid cells in the TMP195-treated mice were highly phagocytic towards the tumor cells, as observed by increased EpCam ${ }^{+}$cells within $\mathrm{F} 4 / 80^{+}$macrophages. Moreover, macrophages in TMP195-treated mice displayed increased expression of stimulating molecules such as CD40 and CD86 and displayed increased antigen presentation. Subsequently, an increase in granzyme $\mathrm{B}^{+}$cells within the cytotoxic $\mathrm{CD}^{+}$ $\mathrm{T}$ cell population was observed. 


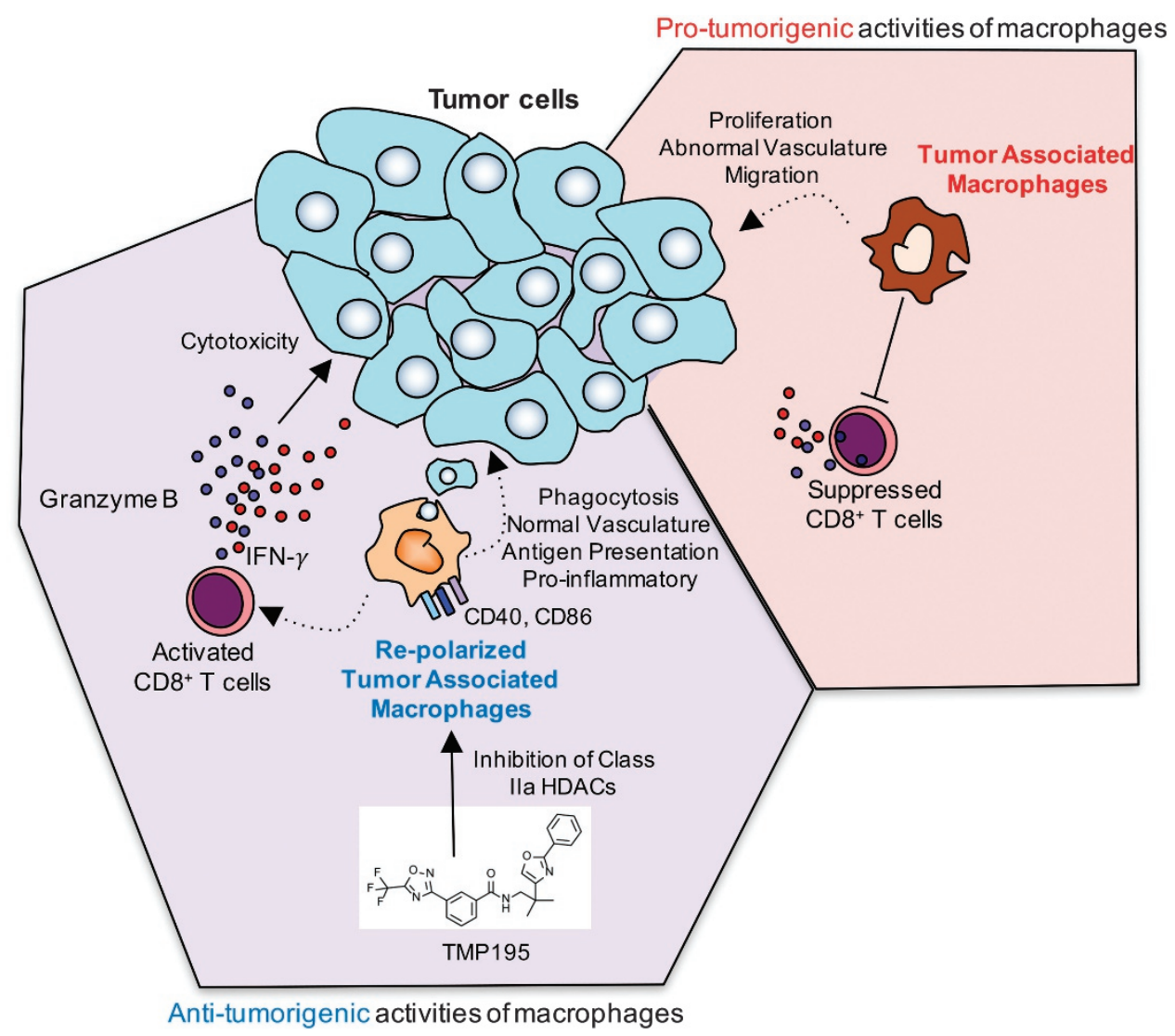

Figure 1 Targeting Class II Histone Deacetylases in Macrophages for Treatment of Breast Cancer. Tumor-associated macrophages (TAMs) are polarized to promote the tumorigenic process. TAMs achieve this through multiple effector pathways. For example, in the MMTV-PyMT macrophage-dependent breast cancer model, TAMs are capable of suppressing CD8 ${ }^{+} \mathrm{T}$ cell cytotoxic activities. They can induce tumor cell proliferation and migration and promote abnormal vasculature (for example, leaky vessel) formation (upper right). Upon treatment with the Class II histone deacetylase inhibitor TMP195 (left side), TAMs become activated and re-polarized to display pro-inflammatory antitumorigenic activities. To this end, they upregulate co-stimulatory molecules such as CD40 and CD86. Moreover, they become highly phagocytic, engulf EpCam ${ }^{+}$tumor cells and are capable of normalizing the vasculature to become less leaky. Upon presentation of tumor antigens to cytotoxic $\mathrm{T}$ cells, the $\mathrm{CD}^{+} \mathrm{T}$ cells likely increase granzyme B expression and increase their ability to kill tumor cells. The tumor vasculature becomes more organized and less leaky. Subsequently, reduction in tumor volume is observed.

Assessment of the blood vessel permeability and leakiness by intravenous injection of labeled heavy dextran demonstrated that the tumor vasculature in TMP195-treated mice was less leaky compared to the untreated group (Figure 1). Macrophage labeling experiments were performed to analyze preexisting vs newly formed macrophages. This analysis showed that the macrophage population that displayed the enhanced phagocytic and immunestimulatory capabilities were newly formed and that TMP195 treatment induced the recruitment of monocytes into the tumor. This result suggests that the inhibition of class IIa HDAC did not induce a phenotypic change in existing macrophages but instead induced the recruitment of monocytes, which differentiated into newly formed macrophages that harbor the potent anti-tumorigenic phenotype.

Ultimately, inhibition of class IIa HDAC caused a marked reduction in the proliferation of tumor cells and resulted in increased tumor cell death. Subsequently, tumor burden in mice treated with TMP195 was reduced compared to that of control vehicle-treated mice. Furthermore, pulmonary metastasis was also markedly decreased, demonstrating an anti-tumorigenic effect for the inhibition of class IIa HDACs. Given that TMP195 treatment did not affect cell death of numerous tumor cell lines in vitro, these effects were most likely indirect and mediated by the ability of TMP195 to re-polarize macrophages (or other $\mathrm{CD}_{11 \mathrm{~b}^{+}}$myeloid cells).

To determine which cells are affected by treatment with TMP195, gene expression in whole tumors was assessed for cell-type signatures. The results from this set of experiments demonstrated that TMP195 predominantly affected monocytes and macrophages. To definitively show that the anti-tumorigenic effect of TMP195 was caused by its activity on macrophages, depletion experiments were performed using anti-CD11b (targeting the entire myeloid population) or anti-CSF1 (specific targeting of macrophages) depleting antibodies. Both of 
these depletion strategies resulted in substantially increased tumor burden in the TMP195-treated mice in comparison to the control antibody treated group, collectively demonstrating that macrophages were required for the antitumorigenic activities of TMP195.

Additional experiments aimed at defining the cellular and molecular downstream events that were triggered by TMP195 treatment demonstrated that depletion of $\mathrm{CD}^{+}$cells or of IFN- $\gamma$ by neutralizing antibodies also abrogated the anti-tumorigenic effect of TMP195. This is an important insight, as it supports the view that, although macrophages are the primary population affected by the TMP195 inhibitor, the anti-tumor effects do not occur solely through direct activities of the macrophages on tumor cells but also require the activation of the adaptive immune response. In fact, these data demonstrate that TMP195 treatment increased macrophage-lymphocyte crosstalk, which provide an additional explanation for the observed increase in tumor cell death.

An emerging concept in the field of immune oncology highlights the potential beneficial effects of combined therapy targeting and enhancing multiple anti-tumorigenic activities. Therefore, Guerriero et $a l^{4}$ examined the effects of
TMP195 treatment in combination with additional treatment strategies that are standard for this model. In combination with chemotherapeutic drugs such as carboplatin (an alkylating agent) or paclitaxel (a plant alkaloid), treatment with TMP195 enhanced the anti-tumor effect compared to therapy with a single agent. Interestingly, while checkpoint blockade using anti-PD-1 is not effective in reducing tumor load in this model, combination of anti-PD-1 and TMP195 was capable of reducing tumor burden and achieving a beneficial effect. Collectively, these data demonstrate that cooperation between innate immune cells and the adaptive immune system leads to a significant anti-tumor response.

This manuscript explores a new approach to anti-tumor therapy in which a selective agent acts on cells from the innate immune system, namely, macrophages, which in turn alter the tumor microenvironment, including cells from the adaptive immune system. Future models could explore other types of cells as potential targets, such as neutrophils and eosinophils, which also harbor the potential to alter the tumor microenvironment in various ways, including modulation of the immune response elicited by cells of the adaptive immune system. $^{7,8}$
CONFLICT OF INTEREST

The authors declare no conflict of interest.

1 Hanahan D, Weinberg RA. Hallmarks of cancer: the next generation. Cell 2011; 144: 646-674.

2 Stromnes IM, Schmitt TM, Chapuis AG, Hingorani SR, Greenberg PD. Re-adapting $T$ cells for cancer therapy: from mouse models to clinical trials. Immunol Rev 2014; 257: 145-164.

3 Noy R, Pollard JW. Tumor-associated macrophages: from mechanisms to therapy. Immunity 2014; 41: 49-61.

4 Guerriero JL, Sotayo A, Ponichtera HE, Castrillon JA, Pourzia AL, Schad S et al. Class Ila HDAC inhibition reduces breast tumours and metastases through antitumour macrophages. Nature 2017; 543: 428-432.

5 Lobera M, Madauss KP, Pohlhaus DT, Wright QG, Trocha M, Schmidt DR et al. Selective class Ila histone deacetylase inhibition via a nonchelating zincbinding group. Nat Chem Biol 2013; 9: 319-325.

6 Guy CT, Cardiff RD, Muller WJ. Induction of mammary tumors by expression of polyomavirus middle T oncogene: a transgenic mouse model for metastatic disease. $\mathrm{Mol} \mathrm{Cell} \mathrm{Biol}$ 1992; 12: 954-961.

7 Reichman H, Karo-Atar D, Munitz A. Emerging roles for eosinophils in the tumor microenvironment. Trends in Cancer 2017; 2: 664-675.

8 Coffelt SB, Wellenstein MD, de Visser KE. Neutrophils in cancer: neutral no more. Nat Rev Cancer 2016; 16: 431-446. 